\title{
LA MECÁNICA DEL HUESO UNA REVISIÓN DE LOS MODELOS DE REMODELACIÓN ÓSEO
}

\author{
Miguel Ángel Flores Rentería, ${ }^{[a]}$ Martín Ortiz Domínguez, ${ }^{[a]}$ Arturo Cruz Avilés ${ }^{[a]}$, Francisco López Sánchez ${ }^{[a]}$ \\ [a1] Profesor Investigador de la Escuela Superior de Ciudad Sahagún de la Licenciatura en Ingeniería Mecánica \\ Universidad Autónoma del Estado de Hidalgo
}

\section{Resumen}

El hueso, al igual que las demás estructuras biológicas se adaptan continuamente a su entorno físico, se ha aceptado ampliamente que el tejido óseo responde a la estimulación mecánica, se reabsorbe en regiones expuestas a estímulos mecánicos de baja intensidad, mientras que se renueva donde estos son altos. Se cree que el proceso de adaptación permite al hueso realizar sus funciones mecánicas con un mínimo de masa. A partir de esta idea muchos modelos teóricos para la remodelación ósea utilizan este concepto como parte de la estrategia para simular la adaptación estructural ósea.

Lo que implican la existencia de un estado de equilibrio donde la estructura ósea se adapta al medio ambiente. Esto ha dado origen al desarrollo de modelos computacionales que, por una parte, suponen una estructura trabecular isotrópica, cuyos resultados muestran las similitudes en la distribución de densidad del hueso in vivo, aun así se considera que no es posible obtener una solución convergente, por otra parte se han desarrollado modelos considerando la naturaleza anisotrópica del hueso trabecular en el nivel continuo haciendo uso de los principios de optimización; Sin embargo, a pesar de las similitudes con el comportamiento real, los modelos, representan simplemente una abstracción matemática de la arquitectura trabecular.

El objetivo de este trabajo es mostrar los modelos de adaptación ósea que emplean una aproximación matemáticos para caracterizar las propiedades mecánicas de la estructura del hueso.

Palabras clave: Hueso, estímulo, remodelado óseo, densidad de energía de deformación

\section{Abstract}

The bone as biological structure is continually adapting to changes in their physical environment, it has been widely accepted that bone respond to mechanical stimulation, tissue is resorbed in regions exposed to low mechanical stimulus, whereas new bone is deposited where the stimulus is high. This process of adaptation is thought to enable bone to perform its mechanical functions with a minimum of mass. From this idea, many theoretical models for bone remodeling use this concept as part of the strategy to simulate bone structural adaptation.

What implies the existence of an equilibrium state where the bone structure is adapted to the environment. That is the origin to develop of computational models were, on one hand, the assumption of isotropy of the trabecular structure is made. The results show the similarities in density distribution with in vivo bone, as well as a convergent solution cannot be obtained. To another part, models have been developed to consider the anisotropic nature of the trabecular bone in the continuum level making use of optimization principles; 
however, despite of some mechanical aspects reflected by these idealized microstructures, they just represent a mathematical abstraction of the trabecular architecture.

The aim of this work is to show the models of bone adaptation that employ a mathematical approach to characterize the mechanical properties of the bone structure.

Keyworks: Bone, stimuli, bone remodeling, strain energy density

\section{Introducción}

El movimiento corporal y la locomoción del cuerpo humano están determinados por la acción de huesos, cartílagos, ligamentos, tendones, músculos y otros tejidos conjuntivos. Los huesos son un sistema dinámico capaz de cambiar su propia densidad, son una estructura pasiva que no genera fuerzas por sí misma, pero está sometida a esfuerzos y deformaciones lo que lo hace responder a diferentes estímulos biomecánicos.

Las funciones principales que desempeñan son: i) proporcionar forma y soporte al cuerpo, ii) proteger a los órganos internos, iii) proveer los puntos de sujeción a la musculatura para generar el movimiento corporal y locomotor, iv) abastecer de minerales, especialmente calcio y fósforo necesarios para mantener el equilibrio entre los líquidos corporales, v) suministrar médula ósea, imprescindible para el desarrollo y almacenamiento de las células sanguíneas. El hueso es un tejido vivo que se encuentra en continuo proceso de crecimiento sujeto a procesos bioquímicos, biofísicos y biológicos relacionados entre sí y con sus propiedades mecánicas y geométricas, (Bruce, Burr, \& Sharkey, 2015).

Generalmente se clasifica con base en su porosidad, por la cual se distinguen dos tipos, el cortical o compacto y el trabecular o esponjoso, el material básico de ambos es el mismo, pero no el grado de porosidad y distribución, las cuales puede cambiar con el tiempo en respuesta a una alteración de cargas, enfermedad o envejecimiento, por lo que el hueso está sometido en forma natural a una involución que, a partir de cierta edad mediante un balance esquelético negativo, lo lleva a una disminución de la masa ósea, para su formación y mantenimiento emplea dos procesos; el modelado y remodelado óseo, el primero involucra el crecimiento y adaptación de los huesos para construir una arquitectura mecánicamente funcional, el segundo consiste en la renovación del hueso, acción que le confiere la capacidad de adaptarse a las cargas mecánicas a las que se le somete permitiéndole variar tanto su estructura interna como externa (Kini \& B, 2012).

Su resistencia depende de su masa, de sus propiedades geométricas, estructurales y materiales (mineralización y composición de la matriz ósea). Las propiedades geométricas y materiales están interrelacionadas mediante un mecanismo sensor retroalimentado. A su vez, las propiedades estructurales están determinadas por las propiedades materiales, por lo que cualquier cambio en las propiedades estructurales debe explicarse con cambios en las propiedades del material.

Es aceptado que el hueso por medio de un mecanismo sensor adapta su estructura a las cargas mecánicas a las que se somete, en este trabajo se aborda la relación de aspectos mecánica y biológicos en los huesos de los seres vivos. Se describe al hueso, el mecanismo sensor encargado de traducir las señales mecánicas en un proceso biológico y aspectos de la remodelación ósea, así como algunos modelos que describen su comportamiento.

\section{El hueso}

Desde el punto de vista macroscópico, se divide en cortical y trabecular, se considera que es rígido y ligero al mismo tiempo. La rigidez la confiere la capa exterior, formada por un material compacto, mientras que en el interior adopta una forma esponjosa que le permite minimizar el peso. Macroscópicamente el tejido esponjoso o trabecular representa alrededor del $80.0 \%$ del volumen total y el cortical aproximadamente el $20.0 \%$, ver figura 1. 


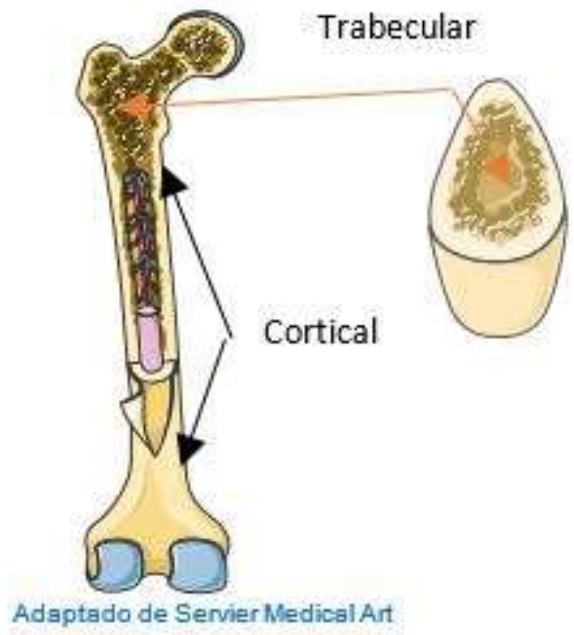

Figura 1. Hueso cortical y Trabecular (Adaptado de Servier Medical Art)

Hueso cortical (relacionado a la corteza), es un tejido sólido que en su interior contiene una red de finos conductos longitudinales (canales de Havers) y transversales (conductos de Volkmann) figura 2, que alojan vasos sanguíneos y fibras nerviosas. El hueso cortical constituye principalmente las diáfisis (parte central) de los huesos largos, la cual adopta una forma cilíndrica para contener la médula ósea. Este tipo de hueso tiene baja porosidad, aproximadamente entre 5 y 10\%. Tiene la característica de ser resistente a la torsión, flexión y al corte (Seeman \& Delmas, 2006).

Hueso trabecular, es un arreglo tridimensional de trabéculas óseas ramificadas, que forman un sistema de espacios intercomunicados. Dentro de cada trabécula se encuentra médula ósea, integrada principalmente por células madre, que producen glóbulos rojos (transportadores de oxigeno) y plaquetas (coaguladores de sangre), además de las células que reciben directamente los nutrientes de la sangre que circula por las cavidades medulares (Osterhof, Morgan, \& Shefelbine, 2016). Este tipo de hueso es la parte metabólica más activa del esqueleto, se encuentra en las epífisis y metáfisis de los huesos largos, constituye la mayor parte del cuerpo vertebral. Contrariamente al cortical, posee una alta porosidad y tiene la capacidad de resistir fuerzas de comprensión y tensión. Se ha considerado que la estructura de las trabéculas está orientada a lo largo de la trayectoria de los esfuerzos. 


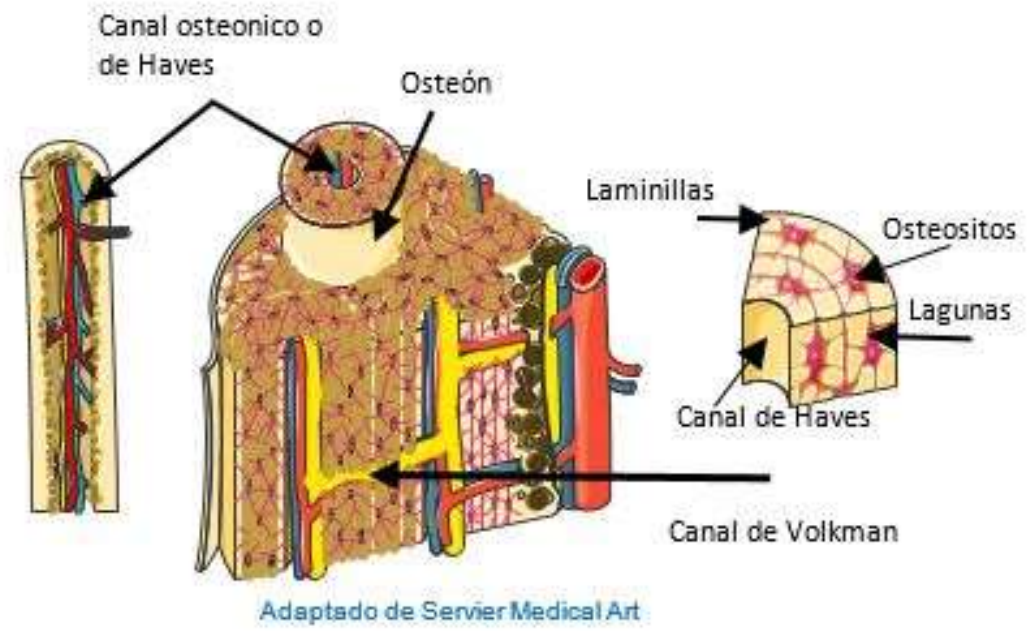

Figura 2. Estructura Ósea (Adaptado de Servier Medical Art).

\section{El mecanismo sensor}

En la literatura se emplea el término mecanobiológico para investigar, principalmente en el tejido óseo, las posibles relaciones entre la estimulación mecánica y la diferenciación células (Mercuri, Daniel, \& Hecke, 2016), (Chowdhury, 2014). El remodelado óseo desde un punto de vista mecanobiológico, implica que las cargas mecánicas que actúan sobre una estructura ósea crean un estímulo biofísico en el tejido, que es detectado por un tipo específico de células. En respuesta a este estímulo, las células excitan moléculas que activan a otras células o alternativamente, actúan ellas mismas para mantener el tejido, remodelarlo o cambiarlo mediante un proceso de diferenciación.

Existe un proceso por el cuál las células óseas son capaces de captar los cambios en la estimulación mecánica de la matriz del hueso para adaptar su arquitectura.

Es ampliamente aceptado que las células óseas son extremadamente sensibles a señales mecánicas, y que el proceso de remodelación ósea se inicia cuando estas reciben una señal emitida por un mecanismo, al cual se le conoce como mecano sensor para iniciar la reabsorción (Cowin, 1991), (Weinans \& R, 1993) (Sommerfeldt \& Rubin, 2001), (Jianying, Haiyan, \& Li, 2007), (Qing \& Wang, 2012), (Gong \& Wang, 2014).

Investigaciones han demostrado, in vitro, que los osteocitos son sensibles a las cargas (Chambers, 1999), Stravos, 2000), (Mercuri \& Daniel, 2016), las cuales activan varios procesos entre los que están el metabolismo de la energía, la activación de genes, los factores de crecimiento y de remodelación. Se ha demostrado que los esfuerzos derivan en un fluido que activa a los osteocitos para producir factores anabólicos, como la prostaglandina y el ácido nítrico. Por lo tanto, es de consenso general que los osteocitos son las células que detectan las señales mecánicas y las transmiten para estimular a los osteoblastos u osteoclastos (Carter, Beaupre, \& Giori, 1998), (Cowin, 1991), (Manolagas, 2000) (Van, Eckstein, \& Koller, 2000), (Gong \& Wang, 2014).

Se ha postulado que la manifestación de los estímulos puede ser la deformación de la laguna osteocita, la densidad de la energía en el tejido óseo (Van, Eckstein, \& Koller, 2000) o la derivación de fluidos en los canalículos osteositarios (Cowin, 1991), (Arden \& H, 1994) .

De modo que las cargas mecánicas tienen una profunda influencia en el remodelado óseo, el mal uso o falta de estas, puede ocasionar un deterioro acelerado del hueso con la reabsorción dominando a la formación y por tanto se tiene una rápida pérdida de masa (Robling et al, 2006).

\section{La remodelación ósea}

El hueso normalmente se encuentra en una constante formación y reabsorción, a lo cual se le conoce como remodelado óseo, es un proceso de reestructuración que permite el mantenimiento del volumen óseo, la 
reparación del daño tisular y la homeostasis del metabolismo fosfocálcico. Este proceso equilibrado, permite la renovación tanto el cortical como el trabecular (Ryser \& Murgas, 2017). Para iniciar la renovación, se cuenta con un mecanismo capaz de detectar y localizar las partes dañadas para removerlas y reemplazarlas con tejido óseo nuevo, restaurando su micro y macro arquitectura (Qing \& Wang, 2012). De modo que no todos los huesos se renuevan a la misma velocidad e incluso en un mismo no es así (por ejemplo, el fémur), pues éste responde a un estímulo de acuerdo con las cargas aplicadas (Seeman \& Delmas, 2006). En el proceso de renovación la reabsorción siempre precede a la formación y en el esqueleto joven las cantidades reabsorbidas son similares a las recién formadas, ver figura 3. Por tal razón se considera un proceso balanceado tanto en el espacio como en el tiempo, mantiene un balance positivo sólo hasta la tercera década en la vida de un individuo, etapa en la cual existe la máxima masa ósea, manteniéndose con pequeñas variaciones hasta los 50 años. A partir de aquí, existe un predominio de la reabsorción y la masa ósea empieza a disminuir. A nivel microscópico el remodelado óseo se produce en pequeñas áreas de la superficie cortical o trabecular, por las llamadas unidades básicas multicelulares (BMU Basic Multicellular Unit), constituidas por osteoblastos, osteoclastos y osteocitos, (Bruce, Burr, \& Sharkey, 2015), donde, por una parte, los osteoclastos reabsorben una cantidad determinada de hueso y por otra, los osteoblastos forman la matriz osteoide para rellenar la cavidad previamente creada, ver figura 3.

En la cual se observa que los osteocitos dentro del fluido óseo detectan la señal de renovación y a través de mediadores activan a los osteoclastos para la iniciar la disolución de la matriz mineral y a descomponer la matriz osteoide, auxiliados por células macrófagos completan la reabsorción liberando factores de crecimiento, que atraen a preosteoblastos para elaborar una sustancia cementante sobre la que se va a adherir el nuevo tejido, tiempo después, los osteoblastos ya diferenciados sintetizan la sustancia osteoide que rellenará las zonas reabsorbidas, en 30 días la sustancia osteoide depositada comienza a mineralizarse. Este proceso tarda aproximadamente 4 mesen en el hueso cortical y 3 en el trabecular. La diferenciación celular es el proceso mediante el cual una célula se convierte en otro tipo celular más especializado. Las BMU están regidas por una serie de factores, tanto generales como locales, permitiendo el normal funcionamiento del hueso y el mantenimiento de la masa ósea. Cuando este proceso se desequilibra aparece la patología ósea, que puede ser por exceso (osteopetrosis) o por defecto (osteoporosis).

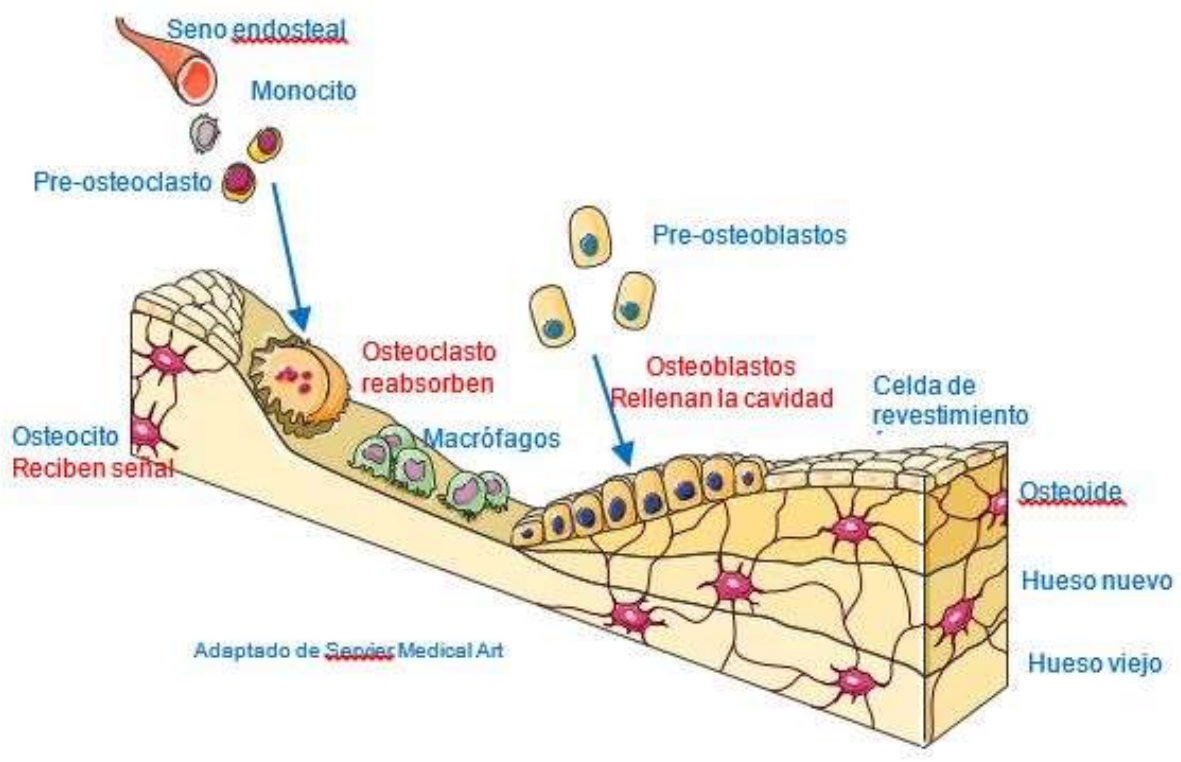

Figura 3. Proceso de remodelación ósea (Adaptado de Servier Medical Art). 
Se han observado diferentes perspectivas del proceso de remodelación ósea, entre ellas está la que considera que este permite optimizar la arquitectura del hueso, para que tenga un mínimo de esfuerzos en su tejido, (Tovar \& Glen, 2014), se toma como base que el cuerpo, en algunos casos remueve hueso, puesto que no se requiere para soportar las cargas y mantiene su masa en un mínimo óptimo, esta consideración requiere que las células óseas estén reguladas por la distribución de esfuerzos en la vecindad inmediata del tejido óseo. Por otra parte, se ha considerado que el proceso de remodelación responde a la acumulación del daño que a diario se genera, debido a las condiciones fisiológicas de carga. Esto sugiere que la remodelación ósea responde a mantener su resistencia, reemplazando al hueso dañado por nuevo para prevenir la acumulación de daños (Badilatti, Christen, \& Parkinson, 2016) el propósito del remodelado es la reparación del hueso, entonces las células deben responder a señales debido al daño que se está presentando.

Uno de los enfoques más aceptado indica que el hueso, por medio del remodelado óseo, se adapta a las condiciones de carga a las cuales es expuesto, lo cual implica que tiene sensores internos que miden las cargas internas traduciéndolas en señales que activan a células capaces de reabsorber o formar hueso, desde hace ya algunos años se ha demostrado como las células pueden detectar las cargas mecánicas y como estas son trasformadas en señales celulares (Ayati, Edwards, \& Webb, 2010), (Ryser \& Murgas, 2017).

Existen evidencias experimentales mostrando que independientemente de los puntos de vista sobre la remodelación ósea, existe un estímulo que incita a la remodelación.

Se ha demostrado de manera experimental que a bajos esfuerzos ocurre una reabsorción y que cuando se incrementan los esfuerzos se da una formación de hueso, células como los osteocitos, implicadas en la remodelación ósea, son capaces de apreciar las deformaciones locales y adaptar la masa de los huesos de acuerdo con las cargas a las que se está sometiendo.

\section{Propiedades mecánicas del hueso.}

El hueso tiene la característica de evolucionar modificando sus propiedades en función de las cargas a las cuales se somete y de regenerarse en caso de sufrir algún daño o de alterar sus propiedades mecánicas ante procesos patológicos o simplemente con la edad.

El hueso es un tejido vivo (su estructura microscópica cambia) que se encuentra en continuo proceso de crecimiento sujeto a procesos bioquímicos, biofísicos y biológicos relacionados entre sí y con las propiedades mecánicas y geométricas.

En el tejido óseo se presentan principalmente dos casos particulares, la isotropía transversal y la ortotropía. La isotropía transversal tiene las características elásticas iguales en dos direcciones perpendiculares, pero diferentes en la tercera, la ortotropía tienen características elásticas diferentes en tres direcciones perpendiculares. El hueso ha sido considerado transversalmente isotrópico (Bruce, Burr, \& Sharkey, 2015) con sus respectivas justificaciones. En la figura 4 se representa la zona cortical de un hueso largo (fémur), el cual tiene una dirección longitudinal definida por el eje del hueso que es perpendicular al plano isotrópico yz. De acuerdo con Dempster y Liddicoat las direcciones transversales xy prácticamente tiene valores iguales y la dirección longitudinal es casi del doble, representando un modelo con isotropía transversal con el plano transversal perpendicular al eje del hueso, plano donde se considera que el comportamiento es isotrópico. 


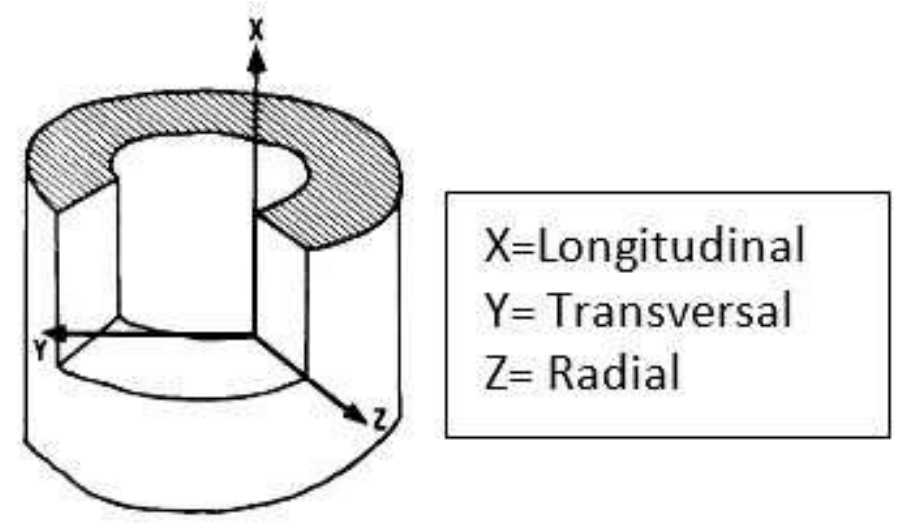

Figura 4 Representación de la isotropía transversal (Adaptado de Servier Medical Art).

Con respecto al hueso trabecular, se sabe que es complejo debido a la heterogeneidad que presenta, un ejemplo se tiene en los extremos del fémur, en la zona trabecular, la cual presenta anisotropía y puede tener un comportamiento similar al de la zona cortical, debido a la orientación que adoptan las trabéculas. La isotropía de la zona trabecular (en los extremos del fémur) dependerá de los esfuerzos que actúen en cada zona del hueso, ya que éstos son los que determinan la orientación o simetría geométrica de las trabéculas. Esta variabilidad en el comportamiento del hueso trabecular ha dado lugar a distintos criterios a la hora de seleccionar el modelo que represente su comportamiento. En muchos estudios el problema se soluciona considerando al hueso como un material isotrópico y en ocasiones como un material ortotrópico.

En la tabla 1 se muestran una serie de valores del módulo de elasticidad tanto para el material cortical como el trabecular para el fémur, que fueron recopilados de los autores citados en este trabajo .

Tabla 1 módulo de elasticidad para el hueso cortical y trabecular en fémur

\begin{tabular}{|l|l|l|l|}
\hline Referencia & Prueba o método & $\begin{array}{l}\text { Cortical } \\
\text { GPa }\end{array}$ & $\begin{array}{l}\text { Trabecular } \\
\text { GPa }\end{array}$ \\
\hline Ashman & Ultrasonido & 20.6 & $12.7 \mathrm{GPa}$. \\
\hline Bernabeu & & $14-20$ & \\
\hline Bilezkian & & 17.0 & 7.6 \\
\hline Burstein & Compresión & & $15.4-18.6$ \\
\hline Burstein & Tensión & $14-20$ & $15.6-17.7$ \\
\hline Gu y Goldstein & & & $14.8+-1.3$ \\
\hline Hackett & & $21.7+-1.2$ & \\
\hline Katsamanis & & 19.9 & \\
\hline Mente & Flexión (E.F) & & $7.8 \mathrm{GPa}$. \\
\hline Meunier & & 20.35 & \\
\hline Wolf & & 20 & $17-20$ \\
\hline Rho & Ultrasonido (tracción) & & $10-14.8$ \\
\hline Rho & Nanoindentación & & $19.6+-3.5$ \\
\hline Reilly & Compresión & & $14.7-19.7$ \\
\hline Reilly & Tensión & & $11.4-19.7$ \\
\hline Turner & & & 20 \\
\hline Yoon & & 27.4 & \\
\hline Zysset & Nanoindentación & & $11.4+-5.6$ \\
\hline
\end{tabular}

El hueso tiene una caracteristica muy especial, posee un mecanismo que detecta los esfuersos mecánicos a los cuales se somete y puede adaptar su estructura interna a la intensidad de las cargas aplicadas incrementando o disminuyendo su volumen (ley de Wolff).

\section{Modelos de la remodelación ósea.}


Aproximadamente desde el siglo dieciocho, la comunidad científica ha estado estudiado la relación existente entre la estructura del tejido óseo y las cargas a las que se encuentra sometido. Se ha observado una relación entre la estructura ósea y la carga a la que se somete, además que el hueso es capaz de adaptarse a las alteraciones que las cargas provoquen (ley de Wolff). Tal consideración ha sido validada y aceptada, mostrando que, a consecuencia de la remodelación ósea, el hueso se adapta a las variaciones de carga.

Desde que inicio el interés por el comportamiento óseo se intentó establecer un modelo constitutivo para la remodelación ósea. Pauwels (1965) propuso la existencia de un estímulo mecánico que controlaba el estado de atrofia o hipertrofia. Sugiere que pequeños valores de tensión provocaban reabsorción, mientras que niveles de tensión mayores implicaban formación. Esta idea se representó matemáticamente por Kummer (1976), pero fueron Cowin y Hegedus (1976) quienes presentan la primera formulación matemática llamada flexibilidad adaptativa, se parte de la hipótesis de que el tejido óseo tiene un estado natural de equilibrio en donde no se produce remodelación ósea. Consideran al hueso como un medio poroso con dos fases: una estructura elástica y un fluido extracelular, la adaptación se presenta mediante una reacción química controlada por la deformación. Emplea la mecánica de medios continuos en la cual las reacciones químicas y la geometría del hueso son forzadas a obedecer, termodinámicamente, principios de masa y momento. El punto de partida del remodelado óseo fue la siguiente ecuación.

$$
\frac{d m}{d t}=c_{i j}\left(\epsilon_{i j}-\epsilon_{i j}^{*}\right)
$$

Donde $c_{i j}$ es un tensor de coeficientes de remodelación, $\epsilon_{i j}$ es el tensor de deformación y $\epsilon_{i j}^{*}$ es una referencia o el tensor de deformación en equilibrio. Con este modelo se mostró que, bajo esfuerzos uniformes en huesos cilíndricos no homogéneos, podrían considerarse homogéneos en lo que a su densidad se refiere (Firoozbakhsh \& Cowin, 1981). Para esfuerzos uniaxiales y ciertos coeficientes de remodelado su modelo es similar al desarrollado por Pauwels. Posteriormente Cowin (1993), presentan una nueva formulación en la que establece que la reorientación y los cambios en el comportamiento anisotrópico de la arquitectura trabecular son función de las tensiones. Para justificar lo anterior introduce el concepto de fabric tensor $\mathrm{H}$, el cual representa a una medida cuantitativa estereológica del reordenamiento microestructural de las trabéculas y los poros del hueso, es decir, caracteriza la microestructura de un material poroso. Además, relaciona este tensor con las constantes elásticas del hueso, obteniendo la primera formulación matemática de la ley de Wolff. Su modelo considera que la condición de equilibrio de remodelación se obtiene cuando las direcciones principales del tejido óseo se encuentran alineadas con las direcciones principales del tensor de deformación que, a su vez, quedará alineado con las del tensor de tensiones. La medida cuantificativa que Cowin introduce se basaba en el porcentaje de volumen sólido del hueso trabecular, el cual variaba entre 0 y 1 y el fabric tensor, el cual es un tensor de segundo orden positivo, con los ejes principales alineados con las direcciones principales de las trabéculas, y cuyos valores son proporcionales a la cantidad de masa de la estructura trabecular asociada a dicha dirección principal. Definió al equilibrio de remodelación por medio de un conjunto de condiciones en las que no se produce reordenamiento de la arquitectura trabecular, ni formación o reabsorción del tejido óseo, planteo dos condiciones de equilibrio: una con la cantidad de volumen del hueso y otra con el reordenamiento anisótropo. Este modelo plantea una formulación complicada puesto que necesita de una gran cantidad de variables para su definición.

Otro punto de vista presentado por Carter (1987) en coincidencia con Frost y Pauwels, es el que considera la adaptación del hueso bajo un concepto de auto optimización en el que un cierto estímulo está presente en el tejido óseo, el cual al mantenerse entre ciertos niveles alcanza un estado cuasi estacionario, el estímulo propuesto era proporcional a una cierta tensión efectiva, la cual consideraba la influencia de un historial de carga durante un periodo de tiempo. La tensión efectiva es una función del estado de tensiones local, correspondiente a cada caso de carga a través de la densidad de energía de deformación y la densidad aparente local, la cual es representada como:

$$
\rho=A \sigma^{\alpha}
$$

$A$ y $\propto$ son constantes, el esfuerzo efectivo está determinado, ya sea, por falla o por un criterio de energía elástica. En publicaciones posteriores (Carter, 1987) determinan que $\propto=0.5 \mathrm{y}$ al esfuerzo efectivo lo determinan de la siguiente forma.

$$
\sigma_{\text {eff }}^{2}=2 E U
$$


$E$ es el módulo de elasticidad aparente y $U$ la densidad de energía de deformación (SED), posteriormente utilizan un módulo de densidad desarrollado por Carter (1977) $E=c p^{3}$, donde c es una constante, la función se transforma en;

$$
\rho=c^{\prime} U
$$

Donde $c^{\prime}$ es constante. Se supone que la remodelación es de la suficiente magnitud para que la funcional de adaptación maximice la rigidez con la menor cantidad ósea. Lo que implicaba considerar a la tensión como una herramienta de optimización que minimiza una función objetivo, a partir de estas consideraciones se propone una relación directa entre la densidad aparente y la tensión efectiva como.

$$
\left.\rho \infty\left(\sum_{i=1}^{N} n_{i} \bar{\sigma}_{i}^{m}\right)^{(1 / 2 m}\right)
$$

Posteriormente este criterio lo utiliza Carter (1987) para determinar la distribución de densidad en el fémur proximal. Para probar la teoría comparan, visualmente, los resultados obtenidos con patrones de radiografías de un fémur real observando similitud con los determinados con la simulación propuesta.

Anteriormente Froest (1964) había sugerido la distinción entre el remodelado interno y el externo. El remodelado interno es la adaptación de la densidad del tejido óseo, mientras que el externo agrega o remueve tejido óseo en la superficie del hueso. Esta consideración fue tomada en cuenta, principalmente por Cowin y Huiskies (1981). Cowin (1985) usan el tensor de deformación como señal mecánica para conducir la adaptación ósea. Firoozbakhsh y Cowin (1981) consideran una relación cuadrática entre la deformación y el porcentaje de adaptación. Huiskies (1987) usa la densidad de energía de deformación (SED $U\left[\frac{\mathrm{J}}{\mathrm{mm}^{3}}\right]$ ) como señal para controlar el remodelado del hueso, para ello definen a $U$ de la siguiente manera.

$$
U=\frac{1}{2} \varepsilon_{i j} \sigma_{i j} \text {. }
$$

La relación entre el porcentaje de adaptación y la SED fue lineal. Para una superficie remodelada externa el hueso puede agregar o remover material de acuerdo con la siguiente expresión.

$$
\frac{d X}{d t}=C_{x}\left(U-U_{n}\right)
$$

Donde $\frac{d X}{d t}$ es el porcentaje de crecimiento en la superficie del hueso, $U$ es la SED, $U_{n}$ es un homeostático de la SED y $C_{x}$ una constante de proporcionalidad. Para el remodelado interno el hueso podría adaptar su valor de densidad. Consideran que el módulo de elasticidad relaciona a la densidad aparente y se escribe la siguiente ecuación.

$$
\frac{d E}{d t}=C_{e}\left(U_{-} U_{n}\right) .
$$

Donde $E[\mathrm{MPa}$ ] es el módulo de elasticidad local y $C e$ una constante de proporcionalidad.

Carter (1984) introduce un el concepto llamado "lazy zone" para indicar que el hueso a la hora de adaptarse o responder al estímulo mecánico es "flojo", e indica que hay una zona de equilibrio, considera que hay un límite en la densidad de energía de deformación el cual se debe de exceder antes de que la adaptación ósea se inicie (ver figura 5), su idea fue incorporada por Huiskes (Huiskes, Weinans, Dalstra, \& Fudala, 1987). La SED es comparada con un homeostático $U_{h}$ para $U>(1+s) U_{h} o U<(1-s) U_{h}$ asociando una actividad adaptativa, por lo cual es el nivel limite el que marca los extremos de la llamada "lazy zone". Con esto se constituye un conjunto de ecuaciones que describen el remodelado del hueso.

$$
\frac{d E}{d t}\left\{\begin{array}{c}
C_{e}\left(U-(1+s) U_{n}\right) \text { para } U>(1+s) U_{n} \\
0 \quad \text { para }(1-s) U_{n} \leq U \leq(1+s) U_{n} \\
C_{e}\left(U-(1-s) U_{n}\right) \text { para } U<(1-s) U_{n}
\end{array}\right\}
$$

Esta teoría se aplicó para predecir la distribución de densidad en fémur normal, comparando los resultados con los obtenidos por Fyhrie y Carter (1986), muestran una similitud, la teoría también fue aplicada para predecir la adaptación cortical después de la adaptación de una prótesis de cadera en un modelo idealizado por Huiskes (1987). 


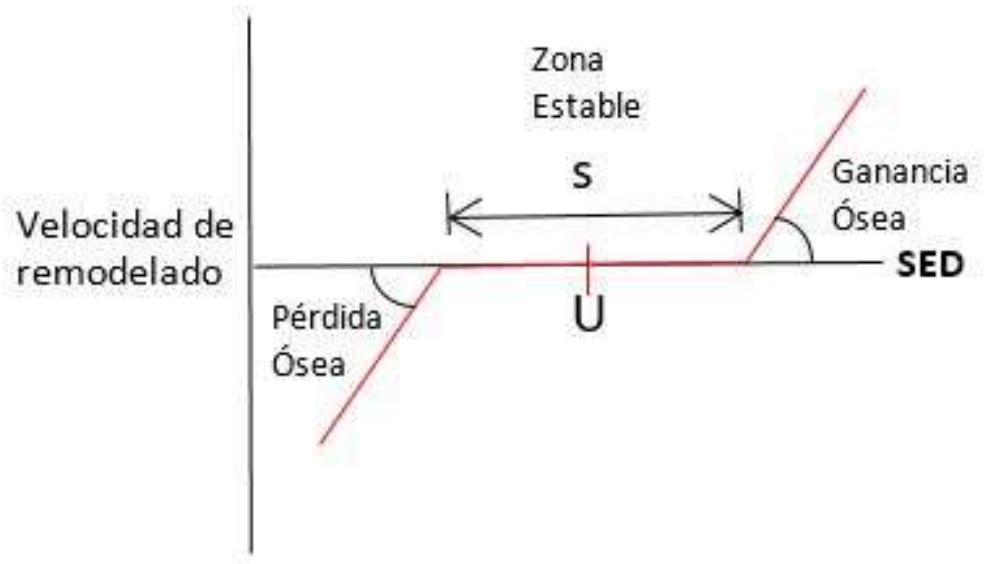

Figura 5 Adaptación local en función de la densidad de energía de adaptación (SED), no hay respuesta adaptativa en la "lazy zone o zona estable" (Adaptado de Huiskes,1987).

En dicho modelo el hueso es representado como un cilindro, se simula el efecto de protección al esfuerzo o stress shielding. La teoría fue aplicada por Rietbergen (Rietbergen, Huiskes, Weinans, Sumner, \& Turn, 1993) para evaluar la adaptación de un fémur después de implantársele una prótesis, el modelo produjo similares resultados de pérdida de hueso a los encontrados experimentalmente en animales.

En las reglas de remodelado expresadas anteriormente, la adaptación del hueso se referencia a estados de deformación causados por cargas simples, para incorporar una carga variable, Carter et al, (1989) definió un estímulo $\mathrm{S}$ que es un promedio de la contribución individual de cargas como.

$$
S=\frac{1}{n} \frac{1}{\rho} \sum_{i=1}^{n} U_{i}
$$

$U_{i}$ es el valor de SED para una carga $i, n$ es el número de casos de cargas y $\rho$ la densidad aparente. Para estimar el valor actual de SED en la trabécula se considera la energía de deformación por unidad de masa $\frac{U}{\rho}$. Esta teoría fue aplicada en un modelo de 2 dimensiones del fémur. El hueso fue representado como un medio continuo y con la capacidad de auto-adaptar su densidad aparente a causa de un estímulo mecánico. El estímulo $S$ fue medido por elemento y la densidad aparente fue adaptada de acuerdo con:

$$
\frac{d \rho}{d t}=B(S-k)
$$

$B$ y $k$ como constantes y $S$ el valor del estímulo.

En las teorías previamente descritas fue necesario incluir su naturaleza biológica, pues la producción de células de remoción ósea no está dentro del hueso, pero aparecen en su superficie como efecto de una señal mecánica, la cual debe ser detectada dentro de la matriz ósea. De modo que Mullender (1994) y (1995) proponen que la detección del estímulo mecánico y la adaptación de las propiedades mecánicas deben estar separadas. Consideran que son los osteocitos mecanismos capaces de detectar las cargas y transmitir las señales a las unidades básicas multicelulares (BMU) encargadas de iniciar la remodelación ósea. La señal enviada por los osteocitos, se supuso que decae exponencialmente con el incremento de la distancia de acuerdo con:

$$
f\left(x, x^{\prime}\right)=e^{\frac{-d\left(x, x^{\prime}\right)}{D}}
$$

Donde $x$ es la ubicación en la cual la señal es determinada, $x^{\prime}$ es la ubicación del osteocito y $D$ determina la decadencia de la señal.

La densidad relativa $m$ en la ubicación $x$ fue regulada por las BMU relativas a la suma total de estímulos $P$ en el lugar que ellos adaptan la densidad de acuerdo con la siguiente expresión: 


$$
\frac{d m(x, t)}{d t}=\tau P(x, t)
$$

En esta ecuación $\tau\left[\mathrm{MPa}^{-1} \mathrm{~S}^{-1}\right]$ es un promedio constante. Los osteocitos localizados en $M$ dentro de una región alrededor de $x$ contribuyen al estimulo de $P$, de modo que su evaluación en el punto $x$ estará determinado por:

$$
P(x, t)=\sum_{i=1}^{N} f\left(x, x_{i}\right)\left(S\left(x_{t}, t\right)-k\right)
$$

$x_{i}$ es la locación del osteocito $i, S\left(x_{i}, t\right)$ es la señal mecánica que el osteocito detecta y $k$ es un valor de referencia.

Posteriormente Huiskes ( $R$, Ruimerman, Lenthe, \& Janssen, 2000) presentas teorías más refinadas, que se basan en un esquema de regulación y en la cuantificación de las relaciones involucradas. La adaptación del tejido en una locación específica $x$ en un tiempo $t$ fue supuesta como resultado de la reabsorción osteoclástica y de la formación osteoblástica, con ello se deduce la siguiente expresión, la cual determina el cambio en la masa ósea en una superficie trabecular.

$$
\frac{d m_{t o t}(x, t)}{d t}=\frac{d m_{c l}(x, t)}{d t}+\frac{d m_{b l}(x, t)}{d t}
$$

Lo que exactamente inicia la reabsorción es desconocido. En la teoría se asume que los osteoclastos son atraídos hacia la superficie del hueso dañado y que estos ocurren de manera aleatoria, de modo que la reabsorción del hueso está determinada de acuerdo a.

$$
\frac{d m_{c l}(x, t)}{d t}=-r_{c l}
$$

Donde $r_{c l}$ [mm3/dia] representa una función estocástica. La actividad osteoblasta fue controlada por una señal de formación osteocita $P$ [mol. $\left.\mathrm{mm}^{-2} \mathrm{dia}^{-1}\right]$. Si el estímulo excede cierto valor limite $k_{t r}\left[\mathrm{~mol}_{\mathrm{mm}} \mathrm{mm}^{-2} \mathrm{dia}^{-1}\right.$ ] entonces la formación del tejido en la superficie trabecular se inicia de acuerdo con la siguiente expresión.

$$
\frac{d m_{b l}(x, t)}{d t}=\tau\left(P(x, t)-k_{t r}\right.
$$

Donde $\tau\left[\mathrm{mm}^{5} \cdot \mathrm{mol}^{-1}\right]$ es factor de proporcionalidad que determina el porcentaje de formación. El estímulo de formación $P$ en la locación $x$ es determinado por todos los osteocitos $N$ localizados dentro de una región de influencia, la señal $R\left[\mathrm{~J} . \mathrm{mm}^{3} . \mathrm{s}^{-1}\right.$ ] sensada por cada osteocito $i$, con una sensitividad $\mu \mathrm{i}\left[\mathrm{mol} . \mathrm{mm} . \mathrm{J}^{-1}\right.$.s.dia ${ }^{-1}$ ] y una localización xi se denota por.

$$
P(x, t)=\sum_{i=1}^{N} f\left(x, x_{i}\right) \mu_{i} R\left(x_{i}, t\right)
$$

En donde $f\left(x, x_{t}\right)$ determina la reducción de la señal de acuerdo con la ecuación 10.

Hasta nuestros días los modelos computacionales del hueso han usado cargas estáticas para evaluar las señales mecánicas. No obstante, se sabe que el hueso responde a cargas dinámicas. En recientes teorías el estímulo detectado por los osteocitos es supuesto como un porcentaje de la densidad de energía de deformación (SEDrate)R(x,t) en una carga reciente. Las condiciones cíclicas de carga caracterizadas por la frecuencia y magnitud fueron impuestas y se consideró que los osteocitos reaccionan en un máximo de la SEDrate durante una carga cíclica y se mostró que el máximo SEDrate está relacionado con el valor de SED, para algún sustito de las cargas estáticas.

Ruimerman considera que los efectos de remodelado bajo cargas dinámicas pueden ser resuelto de la siguiente manera.

Primero considera que los osteocitos detectan, como señal mecánica a la denominada SEDrate, en el reciente historial de cargas. Considera una distribución periódica de un esfuerzo uniforme $\sigma_{e}(t)$ que está variando cíclicamente entre cero y $\sigma[M P a]$ de acuerdo con:

$$
\sigma_{e}(t)=\frac{1}{2} \sigma(1-\cos \omega t)
$$

En donde $\omega=2 \pi f$, con $\mathrm{f}$ como frecuencia $[\mathrm{Hz}]$.

Los esfuerzos externos producen esfuerzos y deformaciones en el interior del hueso en zonas específicas, las cuales son representadas por el esfuerzo principales $\sigma_{i}$ y por las deformaciones principales $\epsilon_{i}(i=1,2,3)$. Al considerar al hueso como material elástico y despreciando los efectos de inercia, los esfuerzos y direcciones principales, son directamente proporcionales a los esfuerzos aplicados externamente y pueden ser escritos como;

$$
\sigma_{i}(t)=\frac{1}{2} c_{i} \sigma(1+\cos \omega t) \text { y por } \epsilon_{i}(t)=\frac{1}{2} e_{i} \sigma(1+\cos \omega t)
$$

En la ecuación anterior $c_{i} y e_{i}$ son los esfuerzos principales y las deformaciones variables independientes del tiempo. Para la densidad de energía de deformación se tiene, por definición, la siguiente expresión.

$$
S(t)=\frac{1}{8} K \sigma^{2}(1+\cos \omega t)^{2}, \quad K=\sum_{i=1}^{3} c_{i} e_{i}
$$


El porcentaje de la densidad de energía de deformación es calculado por;

$$
R(t)=\frac{d s}{d t}=-\frac{K \sigma^{2}}{4}(1+\cos \omega t) \omega \sin \omega t
$$

Anteriormente se consideró que los osteocitos reaccionan con un valor absoluto de la densidad de energía de deformación promedio, la cual ocurre cuando $\omega t=\pi / 3$, su valor puede ser determinado por;

$$
R_{m}=|R(t)|_{\text {max }}=a \sigma^{2} \omega K
$$

Cuando el hueso sea cargado con un esfuerzo constante externo $\sigma^{\prime}$ los esfuerzos y direcciones principales en un punto dado del hueso, serán determiandos por;

$$
\sigma_{i}(t)=c_{i} \sigma^{\prime} \text { y } \epsilon_{i}(t)=e_{i} \sigma^{\prime}
$$

La densidad de energía de deformación se puede calcular por la expresión;

$$
S^{\prime}=1 / 2 K\left[\sigma^{\prime}\right]^{2}
$$

De modo que la densidad de energía de deformación promedio $\mathrm{Rm}$ es igual a la densidad de energía de deformación estática $S^{\prime}$;

$$
\sigma^{\prime}=\sigma(4 a \pi f)^{2} \approx 2 \sigma f^{\frac{1}{2}} \quad \text { con } \quad \omega=2 \pi f
$$

Manuel Doblare (Doblare, 2002) propone un modelo anisotrópico de remodelación ósea, la anisotropía es medida con un fabric tensor normalizado, tal que $\operatorname{det}(\widehat{H})=1$ el tensor se define para considerar la porosidad y la orientación de las porosidades

$$
H(\rho, \widehat{H})=\left(\frac{\rho^{\beta(\rho)} B(\rho)}{\widehat{\rho}^{\left.\beta(\hat{\rho})_{B(}\right)}}\right)^{1 / 4} \widehat{H}^{1 / 2}
$$

En donde $B(\rho)$ y $B(\rho)$ son constantes las cuales depende de $\rho$. El estímulo de activación se representa por $Y$ es un tensor en función de la porosidad y la anisotropía (fabric tensor $H$ ) y del tensor de deformación $€$

$$
Y=2\left[2 \widehat{G} \operatorname{sym}\left[(H \varepsilon H)(H \varepsilon)+\hat{\lambda} \operatorname{tr}\left(H^{2} \varepsilon\right) \operatorname{sym}(H \varepsilon)\right]\right]
$$

$\hat{G} y \hat{\lambda}$ son las constantes de Lamé y $\hat{\rho}$ la densidad del hueso cortical.

Evalúa la evolución de la densidad aparente por medio de $\dot{H}$

$$
\dot{H}=\left\{\begin{array}{lc}
\frac{3 \beta k r \dot{S}_{y}}{4 \operatorname{tr}\left(H^{-2} J^{-3} H \widehat{w}\right)} \frac{\hat{\rho}}{\rho} J^{-3} \widehat{w} & \text { en formación } \\
0 & \text { en reposo } \\
\frac{3 \beta k r \dot{S}_{y}}{4 \operatorname{tr}\left(H^{-2} J^{-3} H \widehat{w}\right)} \frac{\hat{\rho}}{\rho} J \widehat{w} & \text { en reabsorció }
\end{array}\right.
$$

Martínez-Reina (Martínez, García, \& J, 2009) Propone un modelo en el cual considera que la acumulación de daño en la microestructura por fatiga activa a las BMU para remodelar el tejido dañado, pero también se pueden activar para remover al hueso en desuso por medio de un estímulo de magnitud baja. La activación de la BMU depende de aspectos biológicos y de la cantidad de porosidad la cual es modificada por las BMU por medio de una secuencia llamada ARF (Activación-Reabsorción-formación), la duración y el porcentaje de progresión depende de factores fisiológicos y metabólicos.

Este modelo reconoce que el hueso es anisotropico y que las BMU modifican la anisotrópicas al cambiar progresivamente la dirección trabecular, predice la alineación de la micro estructura de acuerdo con las diferentes situaciones de carga aplicada, con el modelo se obtiene las propiedades anisotrópicas y mecánicas de fémur próximas.

\section{Conclusiones}

El hueso es un órgano dinámico, el proceso de remodelación es una reabsorción seguida de la aposición de hueso. Desde hace ya algunas décadas se sabe que el hueso adapta su estructura y masa para soportar los esfuerzos a los que es sometido. Estudios experimentales han mostrado que la densidad del hueso es proporcional a la estimulación mecánica. La actividad mecánica tiene el control de la organización estructural del hueso, sin embargo, el mecanismo por el cual los esfuerzos se traducen en actividad celular para la formación o reabsorción del hueso aun no es del todo entendida. Se han desarrollado un gran número de 
modelos matemáticos, a pesar de esto, los mecanismos que inician y controlan la adaptación ósea no están bien comprendidas y se basan principalmente en hipótesis que todavía necesitan verificación.

Los osteocitos son células que juegan un importante rol en el proceso de adaptación, son considerados como sensores del estímulo mecánico, sin embargo, no hay un acuerdo total sobre el tipo de estímulo mecánico, se han propuesto una variedad en los modelos teóricos como lo son los esfuerzos, la deformación, la razón de deformación, la micro fractura por fatiga, la densidad de energía de deformación, la razón de densidad de energía de deformación.

A pesar de cantidad de modelos y observaciones experimentales sobre el proceso de remodelación ósea quedan muchas preguntas por resolver antes de llegar a un modelo definitivo. La intención de este trabajo es comprender y comparar los modelos más aceptados de remodelación ósea considerando aspecto mecánicos, biológicos y químicos.

\section{Agradecimientos}

Los autores le agradecen al Dr. Jorge Zuno Silva, quien es director de la Escuela Superior de Ciudad Sahagún$U A E H$, México, por todas las facilidades para terminar este trabajo de investigación.

\section{Referencias}

Arden, M., \& H, B. (1994). Function of the osteocytes in bone. Journal of cell and biomechanics, 287-289.

Ayati, B., Edwards, C., \& Webb, G. (2010). A mathematical Model for bone remodeling dynamics for normal bone cell populations and myeloma bone disease. Biology Direct, 2-17.

Badilatti, S., Christen, P., \& Parkinson, I. (2016). Load-adaptive bone remodeling simulations reveal osteoporotic microstructural and mechanical changes in whole human vertebrae. Journal of Biomechanics, 3770-3779.

Bruce, M., Burr, D., \& Sharkey, N. (2015). Skeletal Tissue Mechanics (Segunda ed.). New York: Springer.

Carter, D. (1987). Mechanical loading history and skeletal biology. Journal of Biomechanics, 10951109.

Carter, D. (1987). Trabecular bone density and loading history: regulation of connective tissue biology by mechanical energy. Journal of Biomechanics, 785-794.

Carter, R., Beaupre, G., \& Giori, N. (1998). Mechanobiology of skeletal regeneration. Clinical Orthopaedics, S41-S55.

Chowdhury, B. (2014). Bone Remodeling: The Molecular Mechanism of Bone Physiology-A Review. International Journal Of Scientific CIENTIFIC RESEARCH, 305-306.

Cowin, S. (1991). The mechanical properties of cortical bone tissue. Philadelphia: CRC.

Cowin, S. (1991). The mechanical properties of cortical bone tissue. Philadelphia: CRC.

Cowin, S. (1993). Bone stress adaptation models. Journal of Biomechanical Engineering, 528-533. 
Cowin, S., \& H. H. (1976). Bone remodeling I:Theory of the adaptive elasticity. Journal of elasticity, 313-326.

Doblare, M. G. (2002). Anisotropic bone remodelling model based on a continuum damage-repair theory. Journal of Biomechanics, 1-17.

Firoozbakhsh, K., \& Cowin, S. (1981). An Analytical Model of Pauwels' Functional Adaptation Mechanism in Bone. Journal of Biomechanical Engineering, 246-252.

Gong, H., \& Wang, L. (2014). Computational modeling of bone and bone remodeling. En J. Zhaogmin, Computational Modelling of Biomechanics and Biotribology in the Musculoskeletal System (págs. 244-267). Cambridge: 1.

Huiskes, R., Weinans, H., Dalstra, J. G., \& Fudala, M. (1987). Adaptive bone remodelling theory applied to prosthetic-design analysis. Journal of Biomechanics, 1135-1150.

Jianying, L., Haiyan, L., \& Li, H. (2007). A mathematical model for simulating the bone remodeling process under mechanical stimulus. Dental materials, 1073-1078.

Kini, U., \& B. N. (2012). Physiology of Bone Formation, Remodeling, and Metabolism. En I. Gnanasegaran, \& G. v., Radionuclide and Hybrid Bone Imaging (págs. 29-57). SpringerVerlag.

Kummer, B. (1976). Biomechanics of bone: mechanical properties, functional structure ,functional adaptation. Englewood: Prentice Hall.

M, M., Huiskes, R., \& Weinans, H. (1994). A physiological approach to the simulation of bone remodeling as a self-organizational control process. Journal of Biomechanics, 1389-1394.

Manolagas, S. C. (2000). Birth and Death of Bone Cells. Endocrine Reviews, 115-137.

Martínez, J. R., García, J. A., \& J. D. (2009). A bone remodelling model including the directional activity of BMUs. Biomechanics Model Mechanobiology , 111-127.

Mercuri, E., \& Daniel, A. (2016). Influence of different mechanical stimuli in a multi scale mechanobiological isotropic model for bone remodeling. Medical Engineering and Physics, 904-910.

Mercuri, F., Daniel, A., \& Hecke, M. (2016). Influence of different mechanical stimuli in a milti scale mechanobiological isotropic model for bone remodeling. Medical Engineering and Physics, 904-910.

Mullender, M., \& Huiskes, R. (1995). Proposal for the regulatory mechanism of Wolff's law. Journal of Orthopaedic Research, 503-512.

Osterhof, G., Morgan, E., \& Shefelbine, S. (2016). T he new england journal of medicine. Injury, S11-S20.

Pauwels, F. (1965). Gesammelte abhandlungen zur funktionellen anatomie des bewegungsapparates. Berling: Springer. 
Qing, H. Q., \& Wang, Y. N. (2012). A mathematical model of cortical bone remodeling at cellular level under mechanical stimulus. Acta Mechanica Sinica, 1678-1692.

R, H., Ruimerman, V., Lenthe, H. v., \& Janssen, D. (2000). Effects of mechanical forces on maintenance and adaptation of form in trabecular bone. Nature, 704-706.

Rietbergen, B. v., Huiskes, R., Weinans, H., Sumner, D., \& Turn, M. (1993). The mechanism of bone remodeling and resorption around press-fitted tha stems. Jpurnal of Biomechanics, 369382.

Ryser, M., \& Murgas, K. (2017). Bone remodeling as a spatial evolutionary game. Journal of Theoretical Biology, 16-26.

S, H., \& Carter, D. (1977). The compressive behaviour of bone as a two porous phase structure. The Journal of Bone and Joint Surgery, 954-962.

Seeman, D., \& Delmas, P. (2006). Bone quality, the material and structural basis of bone strength and fragility. The new england journal of medicine, 2250-2261.

Sommerfeldt, D., \& Rubin, C. (2001). Biology of bone a how is orchestrate the form and function of skeleton. European Spine Journal, S86-S95.

Tovar, A., \& G. L. (2014). Hybrid cellular automaton hierarchical algorithm to predict. Journal of Biomechanics, 19-25.

Van, R., Eckstein, F., \& Koller, B. (2000). Trabecular bone tissue strains in the healthy and osteoporotic human femur. 12th Conference of the European Society of Biomechanics,, (págs. 19-29). Dublin.

Weinans, H., \& R, H. (1993). The Behavior of Adaptive Bone-Remodeling Simulation Models. Journa of Biomechanics, 1425-1445. 